\title{
Antioxidant Activity of Extract and Its Major Constituents from Okra Seed on Rat Hepatocytes Injured by Carbon Tetrachloride
}

\author{
Lianmei Hu, ${ }^{1}$ Wenlan Yu, ${ }^{1}$ Ying Li, ${ }^{1}$ Nagendra Prasad, ${ }^{2}$ and Zhaoxin Tang ${ }^{1}$ \\ ${ }^{1}$ College of Veterinary Medicine, South China Agricultural University, Guangzhou 510642, China \\ ${ }^{2}$ Chemical Engineering Discipline, School of Engineering, Monash University, 46150 Bandar Sunway, Malaysia \\ Correspondence should be addressed to Nagendra Prasad; nagendra.prasad@monash.edu \\ and Zhaoxin Tang; zxtangscau@gmail.com
}

Received 2 January 2014; Accepted 16 January 2014; Published 27 February 2014

Academic Editor: José Carlos Tavares Carvalho

Copyright (c) 2014 Lianmei Hu et al. This is an open access article distributed under the Creative Commons Attribution License, which permits unrestricted use, distribution, and reproduction in any medium, provided the original work is properly cited.

The antioxidant activities and protective effects of total phenolic extracts (TPE) and their major components from okra seeds on oxidative stress induced by carbon tetrachloride $\left(\mathrm{CCl}_{4}\right)$ in rat hepatocyte cell line were investigated. The major phenolic compounds were identified as quercetin 3-O-glucosyl $(1 \rightarrow 6)$ glucoside (QDG) and quercetin 3-O-glucoside (QG). TPE, QG, and QDG from okra seeds exhibited excellent reducing power and free radical scavenging capabilities including $\alpha, \alpha$-diphenyl- $\beta$-picrylhydrazyl (DPPH), superoxide anions, and hydroxyl radical. Overall, DPPH radical scavenging activity and reducing power of QG and QDG were higher than those of TPE while superoxide and hydroxyl radical scavenging activities of QG and TPE were higher than those of QDG. Furthermore, TPE, QG, and QDG pretreatments significantly alleviated the cytotoxicity of $\mathrm{CCl}_{4}$ on rat hepatocytes, with attenuated lipid peroxidation, increased SOD and CAT activities, and decreased GPT and GOT activities. The protective effects of TPE and QG on rat hepatocytes were stronger than those of QDG. However, the cytotoxicity of $\mathrm{CCl}_{4}$ on rat hepatocytes was not affected by TPE, QG, and QDG posttreatments. It was suggested that the protective effects of TPE, QG, and QDG on rat hepatocyte against oxidative stress were related to the direct antioxidant capabilities and the induced antioxidant enzymes activities.

\section{Introduction}

Oxidation is essential for living organisms. Reactive oxygen species (ROS) also are produced during oxidation [1]. Organisms can maintain a dynamic equilibrium between production and elimination of ROS in normal conditions. However, when organisms are subjected to stress conditions, this equilibrium is disrupted. Excessive accumulation of ROS will result in cellular injuries, including lipid peroxidation, protein oxidation, and DNA damage, which are involved in development of a variety of diseases including cellular aging, mutagenesis, carcinogenesis, hepatopathies, diabetes, and neurodegeneration [2]. Therefore, cellular antioxidant defense systems play important roles in counteracting these deleterious effects of ROS.

Almost all organisms possess antioxidant defense systems including antioxidant enzymes and nonenzymatic antioxidants. However, these systems are insufficient to prevent the damage entirely in some cases [3]. Plants are the most important source of natural antioxidants [4]. Phenolic compounds or polyphenols, which consist of secondary metabolites, constitute a wide and complex array of phytochemicals that exhibit antioxidant actions. Epidemiological studies have indicated that regular consumption of foods rich in phenolic compounds is associated with reduced risk of cardiovascular diseases, neurodegenerative diseases, and certain cancers [5, 6]. These phenolic compounds hold promising potentials in the development of health foods, nutritional supplements, and herbal medicines for the application as antioxidants and ROS-related disease chemopreventive agents.

Okra (Hibiscus esculentus L.), also known as lady's finger and gumbo, belongs to Malvaceae family, which is distributed widely in Africa, Asia, Southern Europe, and America [7]. The plant is a common vegetable in most regions for its nutrition value. Okra pod contains thick slimy polysaccharides, which are used to thicken soups and stews, 
as an egg white substitute, and as a fat substitute in chocolate bar cookies and in chocolate frozen dairy dessert [7]. Okra seed is rich in protein and unsaturated fatty acids such as linoleic acid [8]. In some countries, okra also is used in folk medicine as antiulcerogenic, gastroprotective, diuretic agents [9]. In addition, Arapitsas [10] reported that okra seed was rich in phenolic compounds, mainly composed of flavonol derivatives and oligomeric catechins, suggesting that it might possess some antioxidant properties. However, little information on antioxidant capabilities of major phenolic compounds from okra seed is available.

Carbon tetrachloride $\left(\mathrm{CCl}_{4}\right)$, a well-known environmental biohazard, can be particularly toxic to liver. $\mathrm{CCl}_{4}$ induced hepatic injury, a classic experimental model, has been extensively used to evaluate the potential of drugs and dietary antioxidants against the oxidative damage [11, 12]. The objectives of the study were to evaluate the antioxidant activity of major phenolic compounds in vitro and their effects on oxidative stress induced by carbon tetrachloride $\left(\mathrm{CCl}_{4}\right)$ in rat hepatocyte cell line.

\section{Materials and Methods}

2.1. Plant Materials. Okra pods (Hibiscus esculentus L.) were harvested from a commercial orchard in Guangzhou, Guangdong, China. The fruit were manually separated, and the seeds were collected, sun-dried, and pulverized to a powder. The materials were stored at room temperature in a desiccator until use.

2.2. Extraction, Isolation, and Purification. Dried seed powder of $H$. esculentus was exhaustively extracted with methanol at temperature $\left(25-32^{\circ} \mathrm{C}\right)$ for 3 days. The extracts were concentrated with a rotary evaporator (RE52AA, Yarong Equipment Co., Shanghai, China) under reduced pressure at $55^{\circ} \mathrm{C}$ and then fractionated sequentially by petroleum ether and EtOAc. The extraction with petroleum ether was to eliminate the pigments. EtOAc extract was obtained by evaporation under reduced pressure and then subjected to purification by silica gel column using $\mathrm{CHCl}_{3}-\mathrm{MeOH}$ solvent system with increased polarity $(0: 100-60: 40)$ to yield eight fractions. We were only interested in the major phenolic compounds. Therefore, the largest fractions were further purified by silica gel column and Sephadex LH-20 to yield compound 1 and compound 2, respectively. Compound 1 and compound 2 were identified as quercetin 3-O-glucosyl $(1 \rightarrow 6)$ glucoside and quercetin 3-O-glucoside (Figure 1), by comparison of experimental and literature NMR data [13].

2.3. Determination of Total Phenol Content. Total phenol content of TPE in H. esculentus was determined by the FolinCiocalteu method [14]. Chlorogenic acid was used as a standard. The total phenol content was determined in triplicate and expressed as chlorogenic acid equivalents in $\mathrm{mg} / \mathrm{g}$ of plant material.

2.4. Evaluation of Antioxidant Activities. Antioxidant capabilities of total phenolic extracts and their major component from okra were evaluated according to the described methods by Duan et al. [15] with minor modifications. To evaluate DPPH scavenging, $0.1 \mathrm{~mL}$ various concentrations of samples were mixed with $2.9 \mathrm{~mL} 0.1 \mathrm{mM}$ DPPH-methanol solution. After $30 \mathrm{~min}$ of incubation at $25^{\circ} \mathrm{C}$ in the dark, the absorbance at $517 \mathrm{~nm}$ was measured. DPPH radical scavenging activity of the samples was calculated using the following formula: DPPH scavenging activity $(\%)=[1-($ absorbance of sampleabsorbance of blank)/absorbance of control] $\times 100$.

Superoxide radicals were generated by illuminating a solution containing riboflavin. The photoinduced reactions were performed at about 4000 lux. $25 \mu \mathrm{L}$ various concentrations of sample were mixed with $3 \mathrm{~mL}$ reaction buffer $[1.3 \mu \mathrm{M}$ riboflavin, $13 \mathrm{mM}$ methionine, $63 \mu \mathrm{M}$ nitroblue tetrazolium (NBT), and $100 \mu \mathrm{M}$ EDTA, pH 7.8]. The reaction solution was illuminated at $25^{\circ} \mathrm{C}$ for $15 \mathrm{~min}$. The reaction mixture without sample was used as a control. The scavenging activity was calculated as follows: scavenging activity $(\%)=(1-$ absorbance of sample/absorbance of control) $\times 100$.

Hydroxyl radical scavenging activity was determined by evaluating inhibitory effect of samples on deoxyribose degradation. $0.2 \mathrm{~mL}$ different concentrations of samples were incubated with $1 \mathrm{~mL}$ reaction buffer $\left(100 \mu \mathrm{M} \mathrm{FeCl}_{3}, 104 \mu \mathrm{M}\right.$ EDTA, $1.5 \mathrm{mM} \mathrm{H}_{2} \mathrm{O}_{2}, 2.5 \mathrm{mM}$ deoxyribose, and $100 \mu \mathrm{M} \mathrm{L}$ ascorbic acid, $\mathrm{pH} 7.4$ ) for $1 \mathrm{~h}$ at $37^{\circ} \mathrm{C}$. After adding $1 \mathrm{~mL}$ $0.5 \% 2$-thiobarbituric acid and $1 \mathrm{~mL} 2.8 \%$ trichloroacetic acid, the mixture was heated for $30 \mathrm{~min}$ at $80^{\circ} \mathrm{C}$ and then cooled on ice. The absorbance at $532 \mathrm{~nm}$ was measured. Percent inhibition of deoxyribose degradation was calculated as (1 absorbance of sample/absorbance of control) $\times 100$.

For the reducing power, $0.2 \mathrm{~mL}$ various concentrations of samples were incubated with $2.0 \mathrm{~mL} 200 \mathrm{mM}$ sodium phosphate buffer ( $\mathrm{pH} 6.6$ ) and $2.0 \mathrm{~mL} \mathrm{1 \%}$ potassium ferricyanide at $50^{\circ} \mathrm{C}$ for $20 \mathrm{~min}$. After adding $2.0 \mathrm{~mL} 10 \%$ trichloroacetic acid $(\mathrm{w} / \mathrm{v})$, the mixture was centrifuged at $4000 \times \mathrm{g}$ for $5 \mathrm{~min}$. $2.0 \mathrm{~mL}$ supernatant was mixed with $2.4 \mathrm{~mL} 0.016 \%$ ferric chloride, and the absorbance at $700 \mathrm{~nm}$ was measured. A higher absorbance indicates a higher reducing power.

2.5. Cell Culture and Cell Treatment. BRL-3A (rat hepatocyte) cell culture was obtained from Experimental Animal Center of Southern Medical University (Guangzhou, China). The cell line was cultured in RPMI-1640 medium containing $10 \%$ foetal bovine serum (FBS), $100 \mathrm{U} / \mathrm{mL}$ penicillin, and $100 \mathrm{mg} / \mathrm{mL}$ streptomycin at $37^{\circ} \mathrm{C}$ in an incubator of humidified air with $5 \% \mathrm{CO}_{2}$. Hepatocytes were seeded onto 24well plates at $1 \times 10^{5}$ cells/well and cultured for $24 \mathrm{~h}$ at $37^{\circ} \mathrm{C}$ under $5 \% \mathrm{CO}_{2}$. Later, the medium was removed and the hepatocytes were subjected to the following treatments: (1) pretreatment, the hepatocytes were first incubated with growth medium containing $100 \mu \mathrm{g} / \mathrm{mL}$ TPE, QG, or QDG for $4 \mathrm{~h}$, and then the cells were washed and incubated with fresh growth medium containing $10 \mathrm{mMCCl}_{4}$ for another $4 \mathrm{~h}$; (2) posttreatment, the hepatocytes were first incubated with growth medium containing $10 \mathrm{mMCCl}_{4}$ for $4 \mathrm{~h}$, and then the cells were washed and incubated with fresh growth medium containing $100 \mu \mathrm{g} / \mathrm{mL}$ TPE, QG, or QDG for another $4 \mathrm{~h}$. 


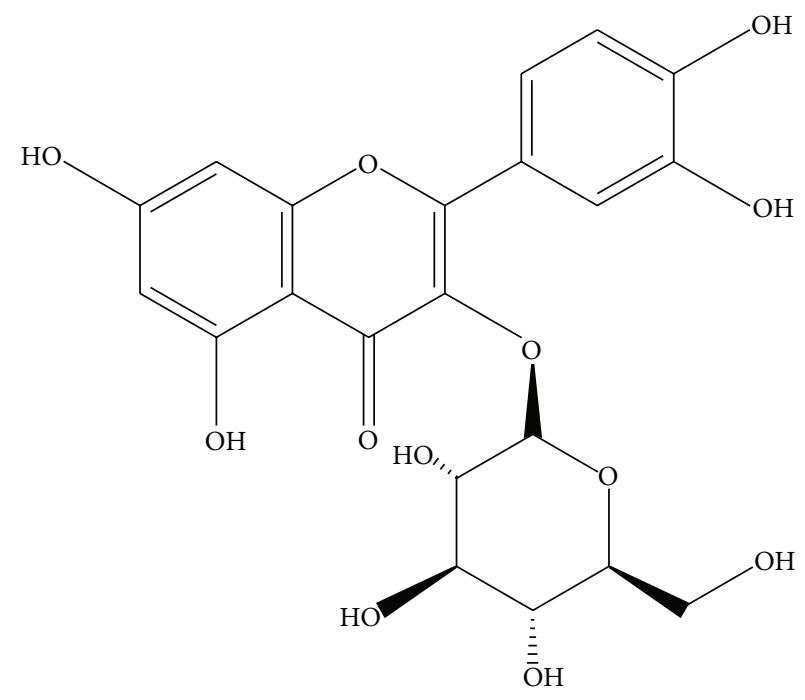

Quercetin 3-O-glucoside

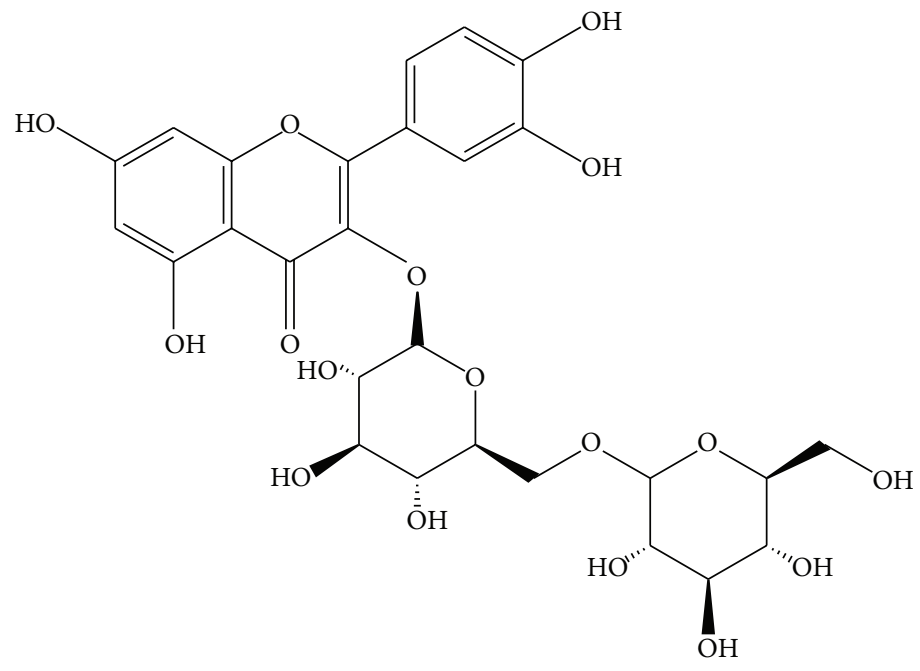

Quercetin 3-O-glucosyl $(1 \rightarrow 6)$ glucoside

Figure 1: The chemical structures of the isolated compounds from Hibiscus esculentus. 1, quercetin 3-O-glucoside; 2, quercetin 3-O-glucosyl $(1 \rightarrow 6)$ glucoside.

2.6. Cell Viability. Cell viability of the hepatocytes was evaluated by MTT assay. After the cells were treated according to the above-mentioned method, the medium was removed and $20 \mu \mathrm{L}$ MTT solution ( $5 \mathrm{~g} / \mathrm{L}$ ) was added to each well of the 96well plate. After $4 \mathrm{~h}$ of incubation at $37^{\circ} \mathrm{C}$, the MTT solution was removed and $100 \mu \mathrm{L}$ dimethylsulphoxide (DMSO) was added to resolve the formazan generated from MTT. The absorbance of each well was recorded on a microplate reader (Thermo, MA, USA) at the wavelength of $490 \mathrm{~nm}$. Cell viability in each test group and model group was expressed as percentage of the control group. The viability of cells in control group was considered as $100 \%$.

2.7. MDA Content and Activities of GPT, GOT, SOD, and $C A T$. After the cells were treated according to the abovementioned method, the supernatants were collected and assayed for malondialdehyde (MDA) content and activities of glutamate pyruvate transaminase (GPT), glutamate oxalate transaminase (GOT), superoxide dismutase (SOD), and catalase (CAT) using commercial enzymatic kits from Nanjing Jiancheng Bioengineering Research Institute (Nanjing, China).

2.8. Statistical Analysis. All data were expressed as mean \pm standard deviation (SD). SPSS was used to analyze and report the data. The differences between the mean values of multiple groups were analyzed by one-way analysis of ANOVA with Duncan's Multiple Range Test. ANOVA data with $P<0.05$ were classified as statistically significant.

\section{Results and Discussions}

3.1. Extraction of TPE and Purification of Major Constituent from Hibiscus esculentus Seed. Hibiscus esculentus 


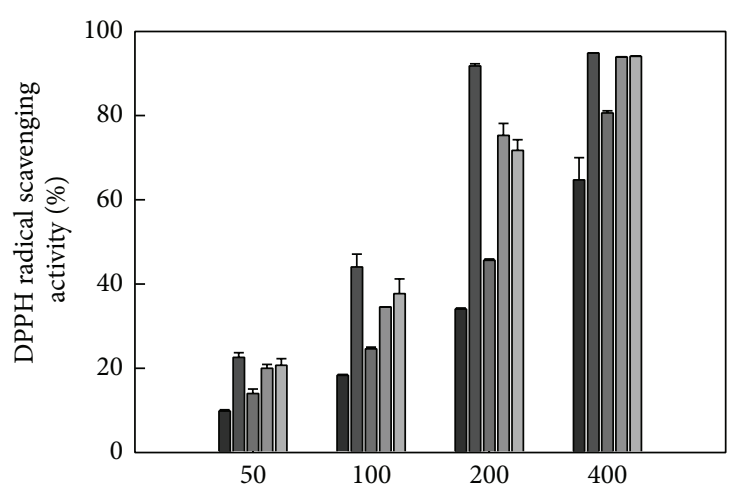

(a)

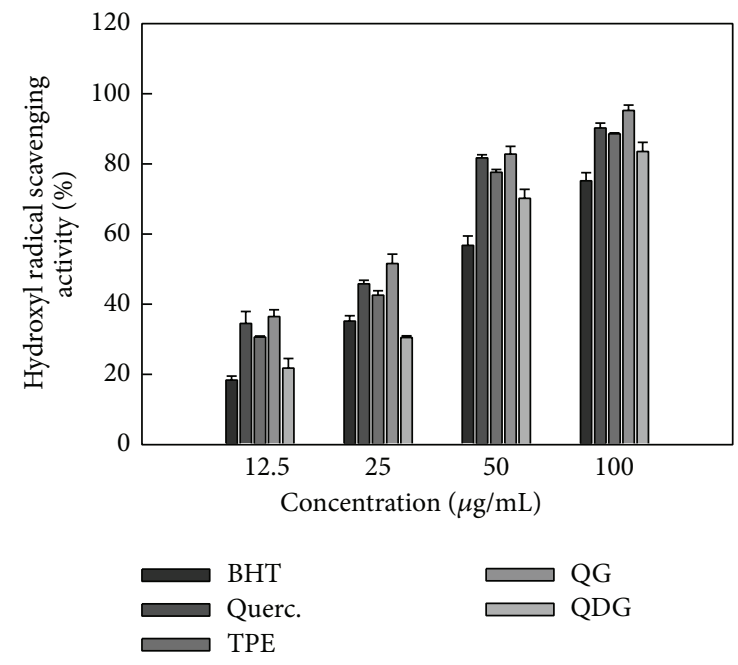

(c)

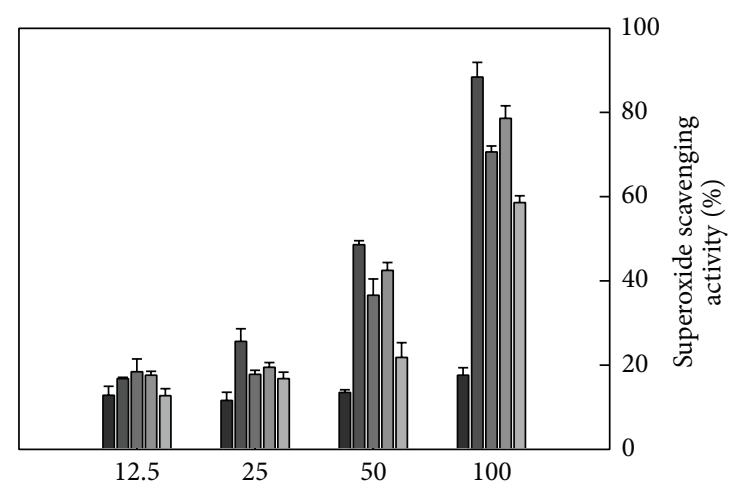

(b)
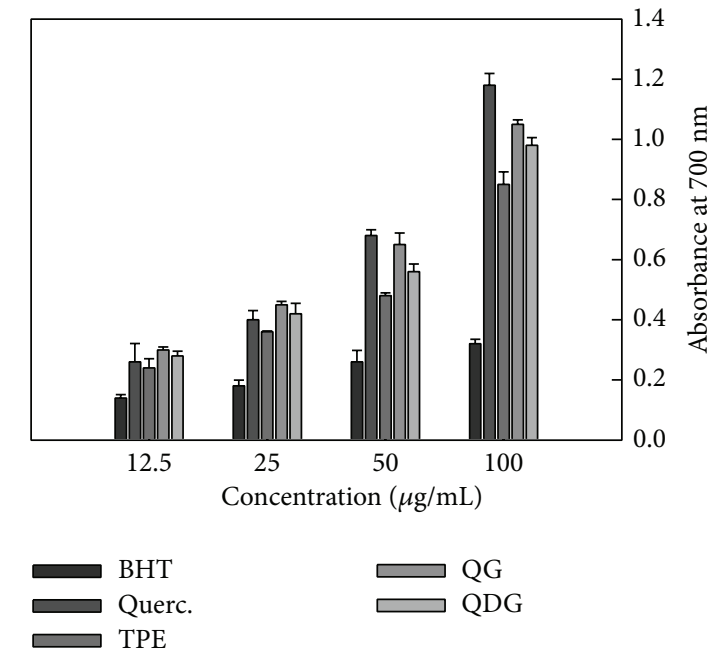

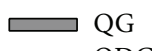

QDG

(d)

FIGURE 2: Free radical scavenging activities against DPPH radical (a), superoxide radical (b), and hydroxyl radical (c) and reducing power (d) of extract and isolated quercetin 3-O-glucoside and quercetin 3-O-diglucoside from Hibiscus esculentus.

seed was subjected to extraction with methanol and then sequential fractionation by petroleum ether and EtOAc. The extraction with petroleum ether was to eliminate the pigments. The EtOAc-soluble fraction was designated as total phenol extracts (TPE). The content of phenolic compounds in dry okra seed was $28.1 \mathrm{mg} / \mathrm{g}$. Further, total phenol extracts (TPE) were purified and two major phenolic compounds were obtained and identified as quercetin 3O-glucosyl $(1 \rightarrow 6)$ glucoside (QDG) and quercetin 3-Oglucoside (QG) (Figure 1), by comparison of experimental and literature NMR data [13], which was consistent with the result reported by Arapitsas [10]. However, Atawodi et al. reported that quercetin glucoside was the only major polyphenol composition in okra seed [16]. The inconsistence might be associated with the differences in climate conditions of cultivation and/or the variety analyzed.

3.2. Antioxidant Activity of Total Phenolic Extracts (TPE) and Their Major Components from Okra Seeds In Vitro. Free radical scavenging activity is the most important mechanism by which antioxidants inhibit lipid peroxidation [1]. In this study, free radical scavenging activity against DPPH radical, superoxide anions, and hydroxyl radical was analyzed to evaluate the antioxidant activities of total phenolic extracts (TPE) and their major components from okra seeds. In addition, reducing power, which exerts antioxidant action by breaking the free radical chain by donating a hydrogen atom, was investigated [15].

DPPH is a stable, purple, nitrogen-centered, and synthetic-free radical with the maximum wavelength of $517 \mathrm{~nm}$. When the DPPH free radical is quenched, the color will fade away. The scavenging activity against DPPH free radical has been extensively used to evaluate antioxidant activity of plant extracts [17]. Figure 2(a) shows the scavenging activities against DPPH free radical of TPE, QG, and QDG from okra seed and BHT and quercetin. All tested samples exhibited the scavenging activities against DPPH free radical in a dose-dependent manner. The positive control quercetin and BHT had the highest and lowest scavenging activity against DPPH free radical, respectively. QG and QDG have almost an equivalent scavenging activity against DPPH free radical, but higher than TPE. At $100 \mu \mathrm{g} / \mathrm{mL}$, the scavenging effects were $18.3 \%, 44.1 \%, 24.6 \%, 34.5 \%$, and $37.3 \%$ for BHT, quercetin, TPE, QG, and QDG, respectively. 
Superoxide anion, produced by a number of cellular reactions or enzymes, including iron-catalyzed Fenton reaction, lipoxygenases, peroxidase, NADPH oxidase, and xanthine oxidase, is the most important in organisms and is involved in the formation of other cell-damaging free radicals [1]. In the present study, TPE, QG, and QDG showed higher scavenging activity against superoxide anion than DPPH radical scavenging activity. Similarly, superoxide anion scavenging activities of TPE, QG, and QDG were stronger than that of BHT, but weaker than that of quercetin. At $50 \mu \mathrm{g} / \mathrm{mL}$ and $100 \mu \mathrm{g} / \mathrm{mL}$ of concentrations, the free radical scavenging activity of TPE was higher than that of QDG, but lower than that of QG (Figure 2(b))

Hydroxyl radical can be formed by the Fenton reaction in the presence of reduced transition metals and $\mathrm{H}_{2} \mathrm{O}_{2}$, which is known to be the most reactive radical and is thought to initiate cell damage in vivo [18]. Similar to superoxide anion scavenging activity, TPE, QG, and QDG from okra seeds exhibited excellent hydroxyl radical scavenging activity in the following order: QG > TPE > QDG > BHT (Figure 2(c)).

Reducing power is widely used to evaluate the antioxidant activity of plant extracts. The reducing property indicates that the antioxidant compounds are electron donors and reduce the oxidized intermediates of lipid peroxidation process [19]. As shown in Figure 2(d), TPE, QG, and QDG from okra seeds showed much higher reducing power than BHT, suggesting that TPE, QG, and QDG possessed a stronger electron donating capacity. However, the reducing power of TPE, QG, and QDG was lower than that of quercetin at $100 \mu \mathrm{g} / \mathrm{mL}$ of concentration. Furthermore, the reducing power of the extract and its major constituents from okra seed were in the following order: QG > QDG > TPE.

Overall, DPPH radical scavenging activity and reducing power of QG and QDG were higher than those of TPE; superoxide and hydroxyl radical scavenging activities of QG and TPE were higher than those of QDG; reducing power, superoxide anion, and hydroxyl radical scavenging activities of QG were higher than those of QDG. Moreover, the antioxidant activities investigated, except hydroxyl radical scavenging activity of quercetin, were higher than those of QG and QDG. It appeared that addition of glycosyl decreased the scavenging activity against DPPH free radical of quercetin and its derivatives. Some similar results were obtained by other researchers. Omololu et al. reported that quercetin had stronger scavenging activity against DPPH free radical than its rhamnosyl glucoside derivative [20]. Hopia and Heinonen investigated the antioxidant activities of quercetin and its selected glycosides in bulk methyl linoleate oxidized at $40^{\circ} \mathrm{C}$ and found that the order of activity of quercetin and its derivatives was quercetin $>$ isoquercitrin $>$ rutin [21]. Sun et al. also found that rhamnosidase could change rutin in asparagus juice to quercetin-3-glucoside, which has a higher antioxidant activity than rutin [22]. The possible reasons are as follows: (1) the steric effect by increased glycosylation decreased the accessibility of free radicals to flavonoid antioxidants; (2) the presence of a free 3-hydroxyl group in the $\mathrm{C}$-ring is a requirement for the maximal radical scavenging activity of flavonoids and the substitution of the hydroxyl group by glycoside resulted in the decreases in free radical

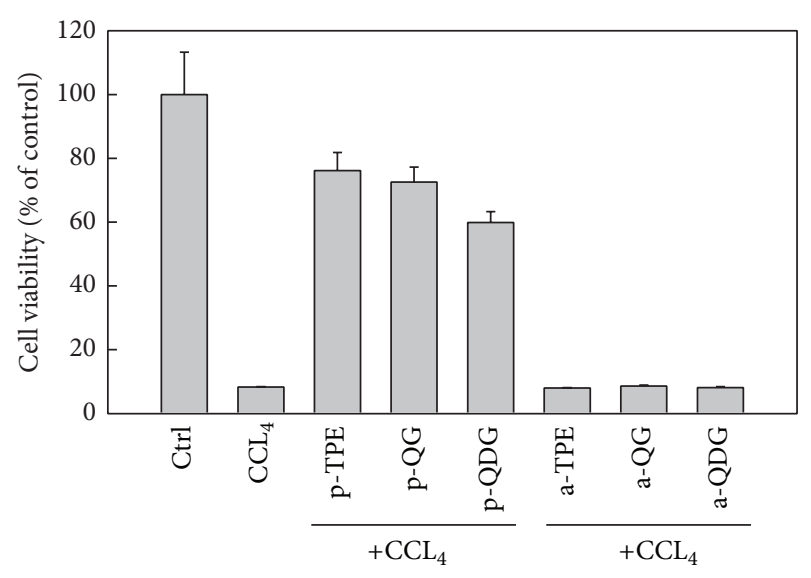

Figure 3: Effect of extract and isolated quercetin 3-O-glucoside and quercetin 3-O-diglucoside from Hibiscus esculentus on $\mathrm{CCl}_{4}$ induced cytotoxicity in rat hepatocytes. In the tick label, $\mathrm{p}$ means pretreatment while a means posttreatment.

scavenging ability and chelating activity to transition metal ions [21, 23]. In addition, QG and QDG played a dominant role in scavenging activity against superoxide anion and hydroxyl radical of total phenolic compounds of okra seed. In some plant flavonoids, quercetin 3-O-glucoside contributed to the major of antioxidant activities [24-27].

3.3. Effect of TPE, QG, and QDG on Cell Viability of Hepatocytes Injured by $\mathrm{CCl}_{4}$. It is well known that cell damage induced by reactive oxygen species (ROS) is an important mechanism of hepatotoxicity [28]. Tetrachloride $\left(\mathrm{CCl}_{4}\right)$ has been widely used to study liver injury induced by ROS in the mouse model. The mechanism is involved in free radicals generated during $\mathrm{CCl}_{4}$ metabolism by hepatic cellular cytochrome $\mathrm{P} 450$, including trichloromethyl $\left(\mathrm{CCl}_{3}\right.$ and/or $\mathrm{CCl}_{3} \mathrm{O}_{2}$ ) and oxygen-centered lipid radicals (LO and/or LOO), which initiate the process of lipid peroxidation [29]. Kikkawa et al. suggested that in vitro primary cell culture system would be sufficient to detect hepatotoxicity in the early stage of drug discovery according to the relevance of in vitro system to in vivo system from some biomarkers related to oxidative stress by carbon tetrachloride [12]. In the present study, $\mathrm{CCl}_{4}$ treatment resulted in a significant decrease in cell viability of the hepatocytes. After $4 \mathrm{~h}$ of incubation, the cell viability decreased to $8.3 \%$ compared with the control, indicating that the hepatocytes were severely injured. TPE, QG, and QDG pretreatments efficiently alleviated oxidative injury of the cell induced by $\mathrm{CCl}_{4}$. The cell viabilities of hepatocytes pretreated with TPE, QG, and QDG were $76.2 \%, 72.5 \%$, and $59.9 \%$, respectively (Figure 3 ). However, posttreatment with TPE, QG, and QDG had no significant protective effect on oxidative injury of the cells caused by $\mathrm{CCl}_{4}$. These results suggest that the extract and its major constituents can be potentially used for preventing rather than curing liver diseases in mammals. A similar result also was reported in fish hepatocytes by Yin et al. [30].

Antioxidants play an important role in protecting against $\mathrm{CCl}_{4}$-induced liver injury. There were some reports on the 
protective effects of various natural products against $\mathrm{CCl}_{4}$ induced liver injury [30-36]. The protective effects might be related to the directed superoxide anion and hydroxyl radical scavenging activity. In our study, superoxide anion and hydroxyl racial scavenging activities were in the following order: QG > TPE > QDG, which was inconsistent with the protective effects. Possibly, the discrepancy could be related to differential uptake by hepatocytes or synergistic effects. Boyer et al. [37] reported that there existed a great difference in uptake of quercetin aglycon and quercetin 3-glucoside as purified compounds and from whole onion and apple peel extracts by Caco- 2 cells. Yang and Liu [38] also found that apple extracts plus quercetin 3-beta-d-glucoside combination possess a synergistic effect in MCF-7 cell proliferation.

\subsection{Effect of TPE, QG, and QDG on Lipid Peroxidation and} Activities of GPT and GOT in Hepatocytes Injured by $\mathrm{CCl}_{4}$. Malondialdehyde (MDA), a lipid peroxidized product, can reflect the extent of lipid peroxidation induced by oxidative stress. As shown in Figure 4, a significant increase in MDA level was observed in the $\mathrm{CCl}_{4}$-treated hepatocytes compared with the control hepatocytes. However, TPE, QG, and QDG treatments at $100 \mu \mathrm{g} / \mathrm{mL}$ significantly decreased the level of lipid peroxidation induced by $\mathrm{CCl}_{4}$. The results were in accordance with the cell viability, indicating that the protective effects of TPE, QG, and QDG on $\mathrm{CCl}_{4}$-induced hepatocytes injury were related to the alleviated lipid peroxidation.

GPT and GOT were widely used to evaluate liver damage by $\mathrm{CCl}_{4}$ [30]. In the present study, significantly elevated GPT and GOT activities were observed in the supernatants of the $\mathrm{CCl}_{4}$-treated hepatocytes, which might be associated with the increased permeability of the hepatocytes and cellular leakage. Pretreatments with TPE, QG, and QDG significantly decreased the values of GPT and GOT activities (Figure 5), indicating that the extract and its major constituents from okra seed could maintain the functional integrity of the hepatocyte membrane, thus protecting the hepatocytes against $\mathrm{CCl}_{4}$-mediated toxicity.

3.5. Effect of TPE, QG, and QDG on Activities of SOD and CAT in Hepatocytes Injured by $\mathrm{CCl}_{4}$. Lipid peroxidation is the result of the excessive accumulation of ROS due to the altered balance between ROS generation and elimination in organism [39]. To control the level of ROS and protect cells against oxidative injury, organisms have developed an enzymatic antioxidant system and low molecular antioxidants [1]. Superoxide dismutase (SOD) is an important defense enzyme that catalyzes the dismutations of superoxide radicals to hydrogen peroxide while catalase (CAT) is involved in eliminating $\mathrm{H}_{2} \mathrm{O}_{2}$. As shown in Figure 6(a), $\mathrm{CCl}_{4}$ treatment alone resulted in a significant decrease in SOD activity of hepatocytes, as compared with the control. However, TPE, QG, and QDG pretreatments restored SOD activities in contrast to the $\mathrm{CCl}_{4}$-treated hepatocytes, which were beneficial in scavenging the superoxide anion and alleviating lipid peroxidation. There were no significant differences in SOD activities among TPE, QG, and QDG pretreated hepatocytes. Moreover, TPE, QG, and QDG posttreatments

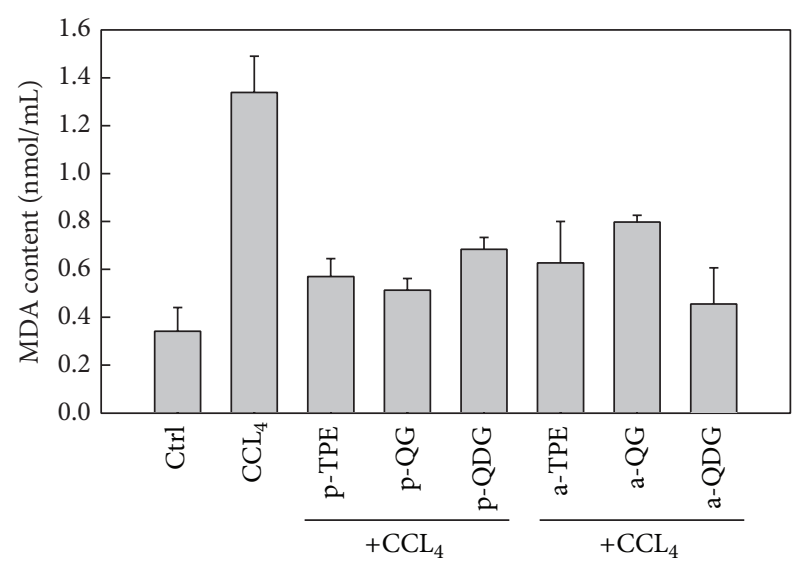

FIGURE 4: Effect of extract and isolated quercetin 3-O-glucoside and quercetin 3-O-diglucoside from Hibiscus esculentus on lipid peroxidation in the cell culture of hepatocytes injured by addition of $\mathrm{CCl}_{4}$ in vitro. In the tick label, $\mathrm{p}$ means pretreatment while a means posttreatment.

also induced SOD activities in the $\mathrm{CCl}_{4}$ pretreated hepatocytes (Figure 6(a)). Similarly, CAT activities in the $\mathrm{CCl}_{4}$ treated hepatocytes also were restored by TPE, QG, and QDG pretreatments. Even the same level of CAT activity, as compared with the control, was found in TPE-treated hepatocytes, which was higher than those in QG and QDG pretreated hepatocytes. CAT activities were partially restored by these treatments (Figure 6(b)).

Possibly, the alleviated biomacromolecule oxidation and elevated cell viability require synergic action of different antioxidant enzymes. There were some reports on the induced activities of antioxidant enzymes by natural products that played an important role in alleviating lipid peroxidation and decreasing the injury caused by $\mathrm{CCl}_{4}[30,31,34,36]$. In the present study, both SOD and CAT activities were induced by pretreatments or posttreatments with TPE, QG, and QDG. However, posttreatment with TPE, QG, and QDG had no significant protective effect on oxidative injury of the cells caused by $\mathrm{CCl}_{4}$. Therefore, it is considered that the induced SOD and CAT activities by posttreatment with TPE, QG, and QDG could not repair the damaged hepatocytes.

\section{Conclusions}

Two major flavonoids, quercetin 3-O-diglucoside (QDG) and quercetin 3-O-glucoside (QG), were isolated and identified from okra seeds. QG, QDG, and the total phenolic extracts (TPE) from okra seeds showed excellent antioxidant activity in vitro, including reducing power and free radical scavenging capabilities against $\alpha, \alpha$-diphenyl- $\beta$-picrylhydrazyl (DPPH) radical, superoxide anion, and hydroxyl radical, which were much stronger than those of BHT, a widely used synthetical antioxidant. DPPH radical scavenging activity and reducing power of QG and QDG were higher than those of TPE while superoxide and hydroxyl radical scavenging activities of QG and TPE were higher than those of QDG. Moreover, reducing power, superoxide anion, and hydroxyl radical 


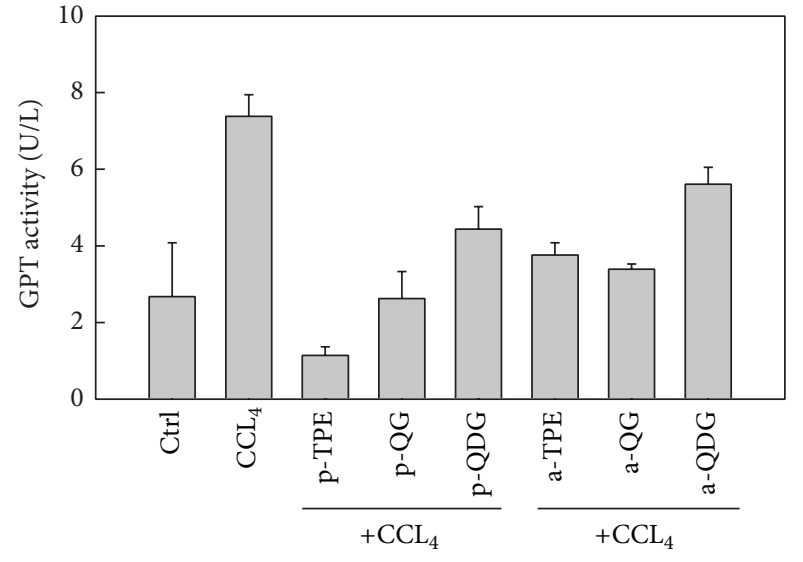

(a)

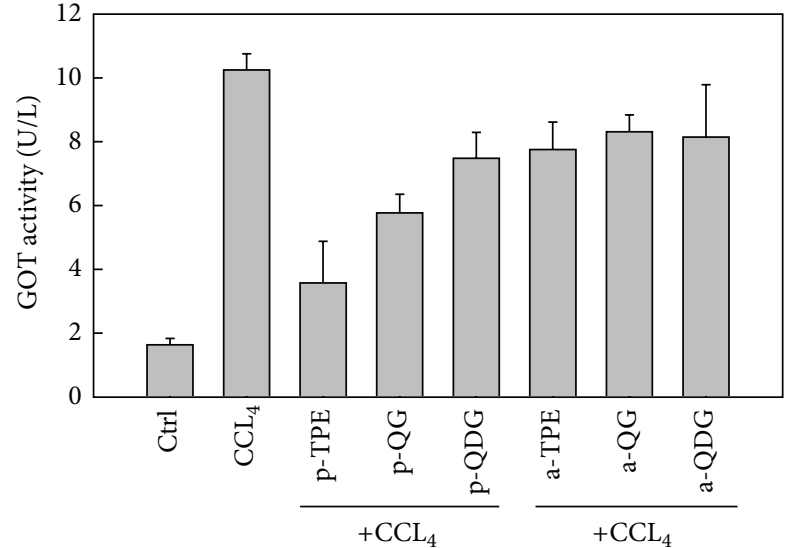

(b)

Figure 5: Effect of extract and isolated quercetin 3-O-glucoside and quercetin 3-O-diglucoside from Hibiscus esculentus on the activities of GPT (a) and GOT (b) in the cell culture of hepatocytes injured by addition of $\mathrm{CCl}_{4}$ in vitro. In the tick label, $\mathrm{p}$ means pretreatment while a means posttreatment.

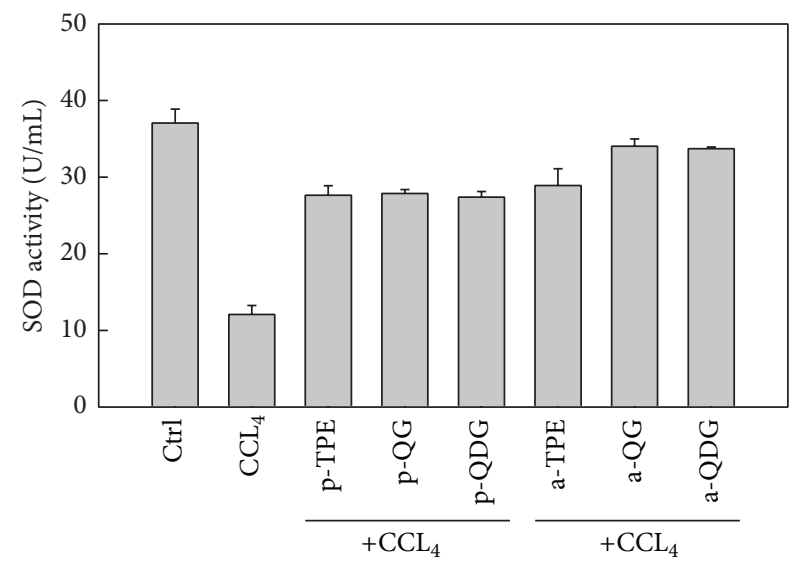

(a)

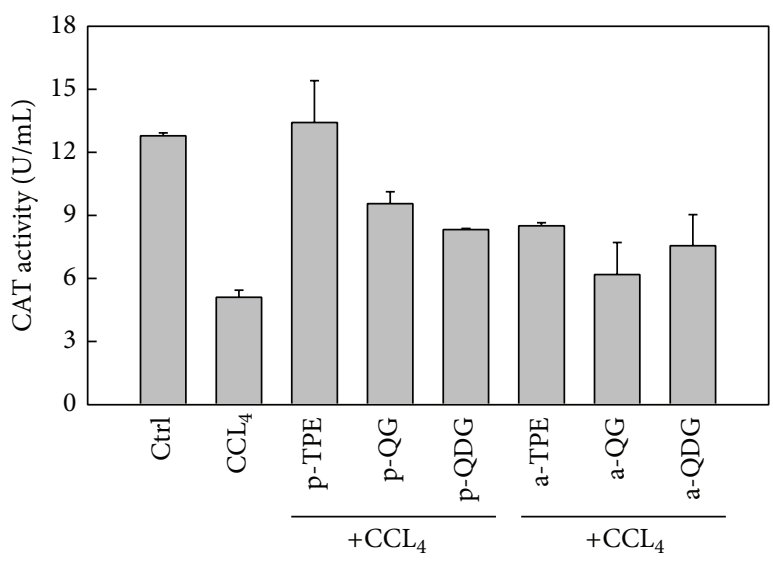

(b)

FIGURE 6: Effect of extract and isolated quercetin 3-O-glucoside and quercetin 3-O-diglucoside from Hibiscus esculentus on the activities of SOD (a) and CAT (b) in the cell culture of hepatocytes injured by addition of $\mathrm{CCl}_{4}$ in vitro. In the tick label, $\mathrm{p}$ means pretreatment while a means posttreatment.

scavenging activities of QG were higher than those of QDG. Furthermore, TPE, QG, and QDG pretreatments significantly alleviated the cytotoxicity of $\mathrm{CCl}_{4}$ on rat hepatocytes, with attenuated lipid peroxidation, increased SOD and CAT activities, and decreased GPT and GOT activities. The protective effects of TPE and QG on rat hepatocytes were stronger than that of QDG. However, the cytotoxicity of $\mathrm{CCl}_{4}$ on rat hepatocytes was not affected by TPE, QG, and QDG posttreatments. It was suggested that the protective effects of TPE, QG, and QDG on rat hepatocyte against oxidative stress were related to the direct antioxidant activity and the induced activities of antioxidant enzymes. However, further investigation is required to evaluate protective effects of total phenolic extracts (TPE) and their major components from okra seeds on carbon tetrachloride-induced hepatotoxicity in vivo.

\section{Conflict of Interests}

The authors declare that there is no conflict of interests regarding the publication of this paper.

\section{Authors' Contribution}

Lianmei Hu and Wenlan Yu contributed equally to this work.

\section{Acknowledgments}

This work was supported by Science and Technology Planning Project of Guangdong Province, China (Grant no. 2011B020306009), the Knowledge Innovation Program of the Chinese Academy of Sciences (Grant no. KSCX2-EW-Q-8-1), and National Natural Science Foundation of China (Grant no. 31171778). 


\section{References}

[1] O. Blokhina, E. Virolainen, and K. V. Fagerstedt, "Antioxidants, oxidative damage and oxygen deprivation stress: a review," Annals of Botany, vol. 91, no. 2, pp. 179-194, 2003.

[2] J. Moskovitz, M. B. Yim, and P. B. Chock, "Free radicals and disease," Archives of Biochemistry and Biophysics, vol. 397, no. 2, pp. 354-359, 2002.

[3] M. G. Simic, "Mechanisms of inhibition of free-radical processes in mutagenesis and carcinogenesis," Mutation Research, vol. 202, no. 2, pp. 377-386, 1988.

[4] K. N. Prasad, H. H. Xie, J. Hao et al., "Antioxidant and anticancer activities of 8-hydroxypsoralen isolated from wampee [Clausena lansium (Lour.) Skeels] peel," Food Chemistry, vol. 118 , no. 1, pp. 62-66, 2010.

[5] M. Soory, "Nutritional antioxidants and their applications in cardiometabolic diseases," Infectious Disorders, vol. 12, no. 5, pp. 388-401, 2012.

[6] H. Y. Aboul-Enein, P. Berczynski, and I. Kruk, "Phenolic compounds: the role of redox regulation in neurodegenerative disease and cancer," Mini-Reviews in Medicinal Chemistry, vol. 13, no. 3, pp. 385-398, 2013.

[7] N. Sengkhamparn, R. Verhoef, H. A. Schols, T. Sajjaanantakul, and A. G. J. Voragen, "Characterisation of cell wall polysaccharides from okra (Abelmoschus esculentus (L.) Moench)," Carbohydrate Research, vol. 344, no. 14, pp. 1824-1832, 2009.

[8] O. J. Oyelade, B. I. O. Ade-Omowaye, and V. F. Adeomi, "Influence of variety on protein, fat contents and some physical characteristics of okra seeds," Journal of Food Engineering, vol. 57, no. 2, pp. 111-114, 2003.

[9] I. Gürbüz, O. Üstün, E. Yesilada, E. Sezik, and O. Kutsal, "Antiulcerogenic activity of some plants used as folk remedy in Turkey," Journal of Ethnopharmacology, vol. 88, no. 1, pp. 93-97, 2003.

[10] P. Arapitsas, "Identification and quantification of polyphenolic compounds from okra seeds and skins," Food Chemistry, vol. 110, no. 4, pp. 1041-1045, 2008.

[11] S. Basu, "Carbon tetrachloride-induced lipid peroxidation: eicosanoid formation and their regulation by antioxidant nutrients," Toxicology, vol. 189, no. 1-2, pp. 113-127, 2003.

[12] R. Kikkawa, M. Fujikawa, T. Yamamoto, Y. Hamada, H. Yamada, and I. Horii, "In vivo hepatotoxicity study of rats in comparison with in vitro hepatotoxicity screening system," The Journal of Toxicological Sciences, vol. 31, no. 1, pp. 23-34, 2006.

[13] G. H. Shui and L. L. Peng, "An improved method for the analysis of major antioxidants of Hibiscus esculentus Linn," Journal of Chromatography A, vol. 1048, no. 1, pp. 17-24, 2004.

[14] V. L. Singleton and J. A. Rossi, "Colorimetry of total phenolics with phosphomolybdic-phosphotungstic acid reagents," American Journal of Enology and Viticulture, vol. 16, no. 3, pp. 144-158, 1965.

[15] X. W. Duan, Y. M. Jiang, X. G. Su, Z. Q. Zhang, and J. Shi, "Antioxidant properties of anthocyanins extracted from litchi (Litchi chinenesis Sonn.) fruit pericarp tissues in relation to their role in the pericarp browning," Food Chemistry, vol. 101, no. 4, pp. 1365-1371, 2007.

[16] S. E. Atawodi, J. C. Atawodi, G. A. Idakwo et al., "Polyphenol composition and antioxidant potential of Hibiscus esculentus L. fruit cultivated in Nigeria," Journal of Medicinal Food, vol. 12, no. 6, pp. 1316-1320, 2009.

[17] C. Sánchez-Moreno, "Review: methods used to evaluate the free radical scavenging activity in foods and biological systems,"
Food Science and Technology International, vol. 8, no. 3, pp. 121$137,2002$.

[18] E. Rollet-Labelle, M.-J. Grange, C. Elbim, C. Marquetty, M.A. Gougerot-Pocidalo, and C. Pasquier, "Hydroxyl radical as a potential intracellular mediator of polymorphonuclear neutrophil apoptosis," Free Radical Biology and Medicine, vol. 24, no. 4, pp. 563-572, 1998.

[19] K. H. S. Farvin and C. Jacobsen, "Phenolic compounds and antioxidant activities of selected species of seaweeds from Danish coast," Food Chemistry, vol. 138, no. 2-3, pp. 1670-1681, 2013.

[20] P. A. Omololu, J. B. T. Rocha, and I. J. Kade, "Attachment of rhamnosyl glucoside on quercetin confers potent iron-chelating ability on its antioxidant properties," Experimental and Toxicologic Pathology, vol. 63, no. 3, pp. 249-255, 2011.

[21] A. Hopia and M. Heinonen, "Antioxidant activity of flavonol aglycones and their glycosides in methyl linoleate," Journal of the American Oil Chemists' Society, vol. 76, no. 1, pp. 139-144, 1999.

[22] T. Sun, J. R. Powers, and J. Tang, "Enzyme-catalyzed change of antioxidants content and antioxidant activity of asparagus juice," Journal of Agricultural and Food Chemistry, vol. 55, no. 1, pp. 56-60, 2007.

[23] K. E. Heim, A. R. Tagliaferro, and D. J. Bobilya, "Flavonoid antioxidants: chemistry, metabolism and structure-activity relationships," The Journal of Nutritional Biochemistry, vol. 13, no. 10, pp. 572-584, 2002.

[24] M. J. Piao, E. S. Yoo, Y. S. Koh et al., "Antioxidant effects of the ethanol extract from flower of Camellia japonica via scavenging of reactive oxygen species and induction of antioxidant enzymes," International Journal of Molecular Sciences, vol. 12, no. 4, pp. 2618-2630, 2011.

[25] M. de Nisco, M. Manfra, A. Bolognese et al., "Nutraceutical properties and polyphenolic profile of berry skin and wine of Vitis vinifera L. (cv. Aglianico)," Food Chemistry, vol. 140, no. 4, pp. 623-629, 2013.

[26] R. Garcia-Mateos, L. Aguilar-Santelises, M. Soto-Hernandez, and R. Nieto-Angel, "Flavonoids and antioxidant activity of flowers of Mexican Crataegus spp.", Natural Product Research, vol. 27, no. 9, pp. 834-836, 2013.

[27] A. M. Mendoza-Wilson, M. E. Armenta-Vazquez, S. I. CastroArredondo et al., "Potential of polyphenols from an aqueous extract of apple peel as inhibitors of free radicals: an experimental and computational study," Journal of Molecular Structure, vol. 1035, pp. 61-68, 2013.

[28] E. A. Ferreira, E. F. Gris, K. B. Felipe et al., "Potent hepatoprotective effect in $\mathrm{CCl}_{4}$-induced hepatic injury in mice of phloroacetophenone from Myrcia multiflora," Libyan Journal of Medicine, vol. 5, no. 1, 2010.

[29] X.-W. Li, R. Zhu, B. Li et al., "Mechanism underlying carbon tetrachloride-inhibited protein synthesis in liver," World Journal of Gastroenterology, vol. 16, no. 31, pp. 3950-3956, 2010.

[30] G. J. Yin, L. P. Cao, P. Xu, G. Jeney, and M. Nakao, "Hepatoprotective and antioxidant effects of Hibiscus sabdariffa extract against carbon tetrachloride-induced hepatocyte damage in Cyprinus carpio," In Vitro Cellular and Developmental Biology, vol. 47, no. 1, pp. 10-15, 2011.

[31] Y. Wu, L. Li, T. Wen, and Y.-Q. Li, "Protective effects of echinacoside on carbon tetrachloride-induced hepatotoxicity in rats," Toxicology, vol. 232, no. 1-2, pp. 50-56, 2007.

[32] J.-Y. Zhong, H.-Q. Cong, and L.-H. Zhang, "Inhibitory effects of grape procyanidins on free radical-induced cell damage in 
rat hepatocytes in vitro," World Journal of Gastroenterology, vol. 13, no. 19, pp. 2752-2755, 2007.

[33] J.-F. Ye, H. Zhu, Z.-F. Zhou et al., "Protective mechanism of andrographolide against carbon tetrachloride- induced acute liver injury in mice," Biological and Pharmaceutical Bulletin, vol. 34, no. 11, pp. 1666-1670, 2011.

[34] S. Ilavenil, B. Kaleeswaran, and S. Ravikumar, "Protective effects of lycorine against carbon tetrachloride induced hepatotoxicity in Swiss albino mice," Fundamental \& Clinical Pharmacology, vol. 26, no. 3, pp. 393-401, 2012.

[35] W. Jiang, M. Gao, S. A. Sun et al., "Protective effect of L-theanine on carbon tetrachloride-induced acute liver injury in mice," Biochemical and Biophysical Research Communications, vol. 422, no. 2, pp. 344-350, 2012.

[36] S. Zhang, B. N. Lu, X. Han et al., "Protection of the flavonoid fraction from Rosa laevigata Michx fruit against carbon tetrachloride-induced acute liver injury in mice," Food and Chemical Toxicology, vol. 55, pp. 60-69, 2013.

[37] J. Boyer, D. Brown, and H. L. Rui, "Uptake of quercetin and quercetin 3-glucoside from whole onion and apple peel extracts by Caco-2 cell monolayers," Journal of Agricultural and Food Chemistry, vol. 52, no. 23, pp. 7172-7179, 2004.

[38] J. Yang and R. H. Liu, "Synergistic effect of apple extracts and quercetin $3-\beta$-D-glucoside combination on antiproliferative activity in MCF-7 human breast cancer cells in vitro," Journal of Agricultural and Food Chemistry, vol. 57, no. 18, pp. 8581-8586, 2009.

[39] D. Trachootham, W. Lu, M. A. Ogasawara, N. R.-D. Valle, and P. Huang, "Redox regulation of cell survival," Antioxidants \& Redox Signaling, vol. 10, no. 8, pp. 1343-1374, 2008. 

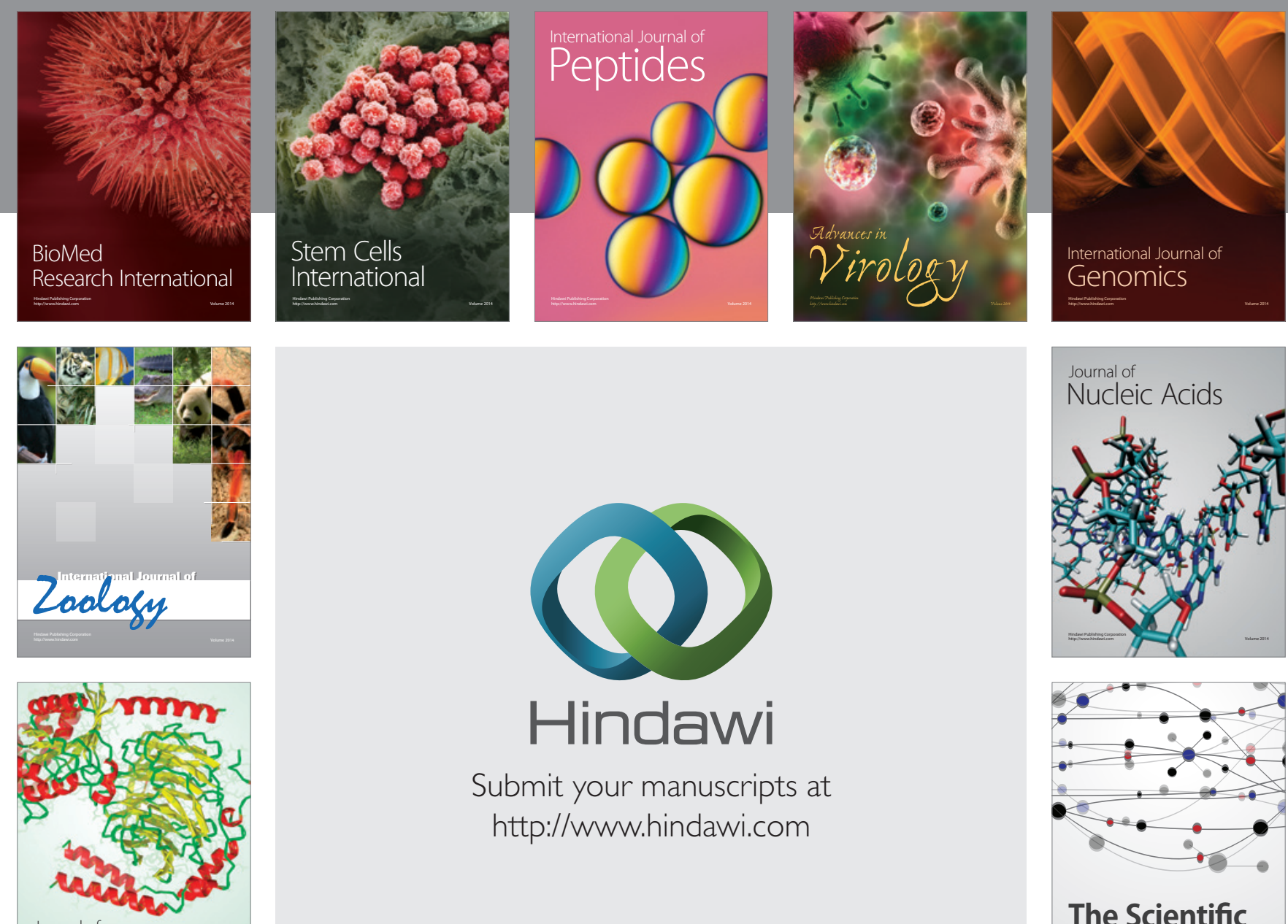

Submit your manuscripts at

http://www.hindawi.com

Journal of
Signal Transduction
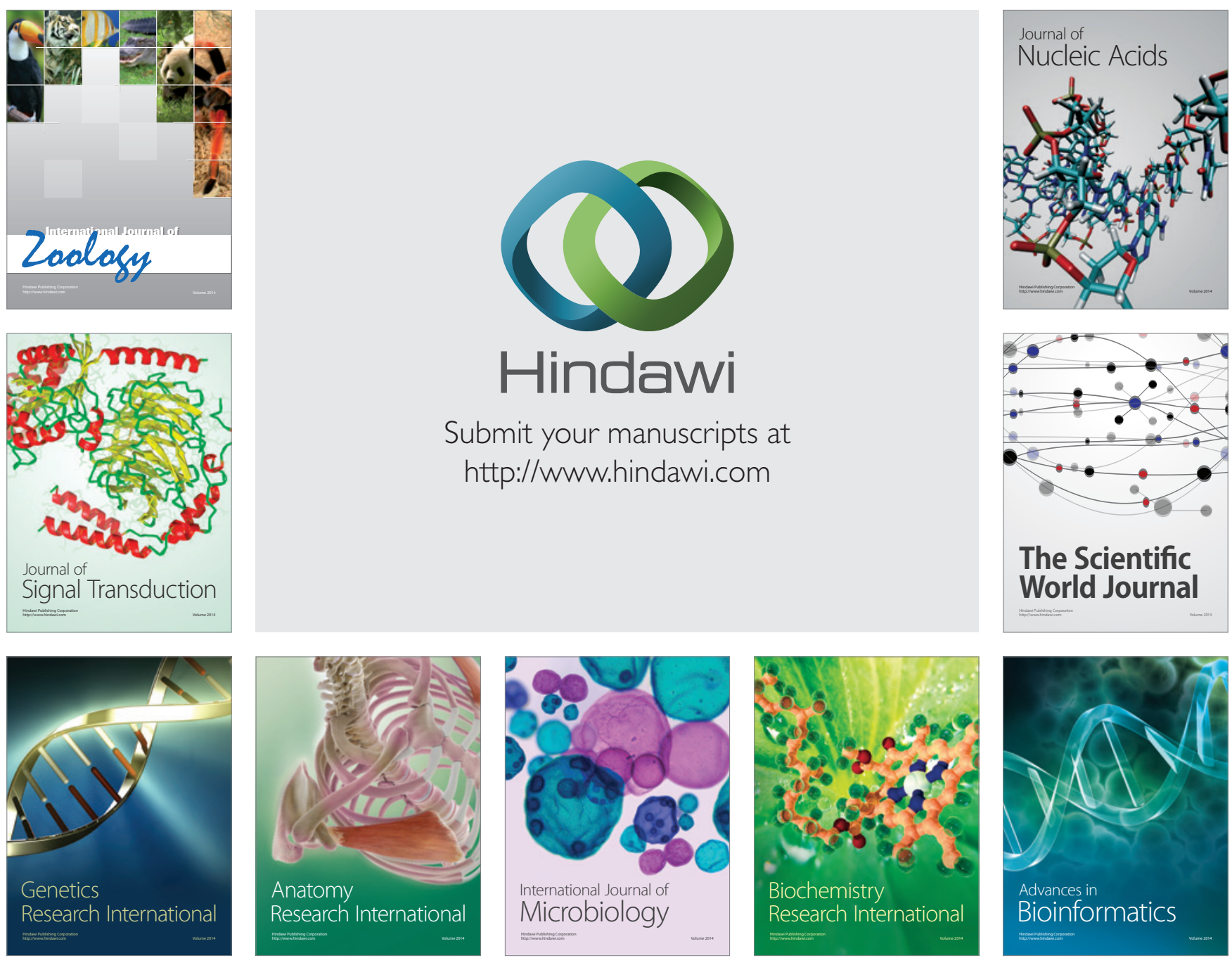

The Scientific World Journal
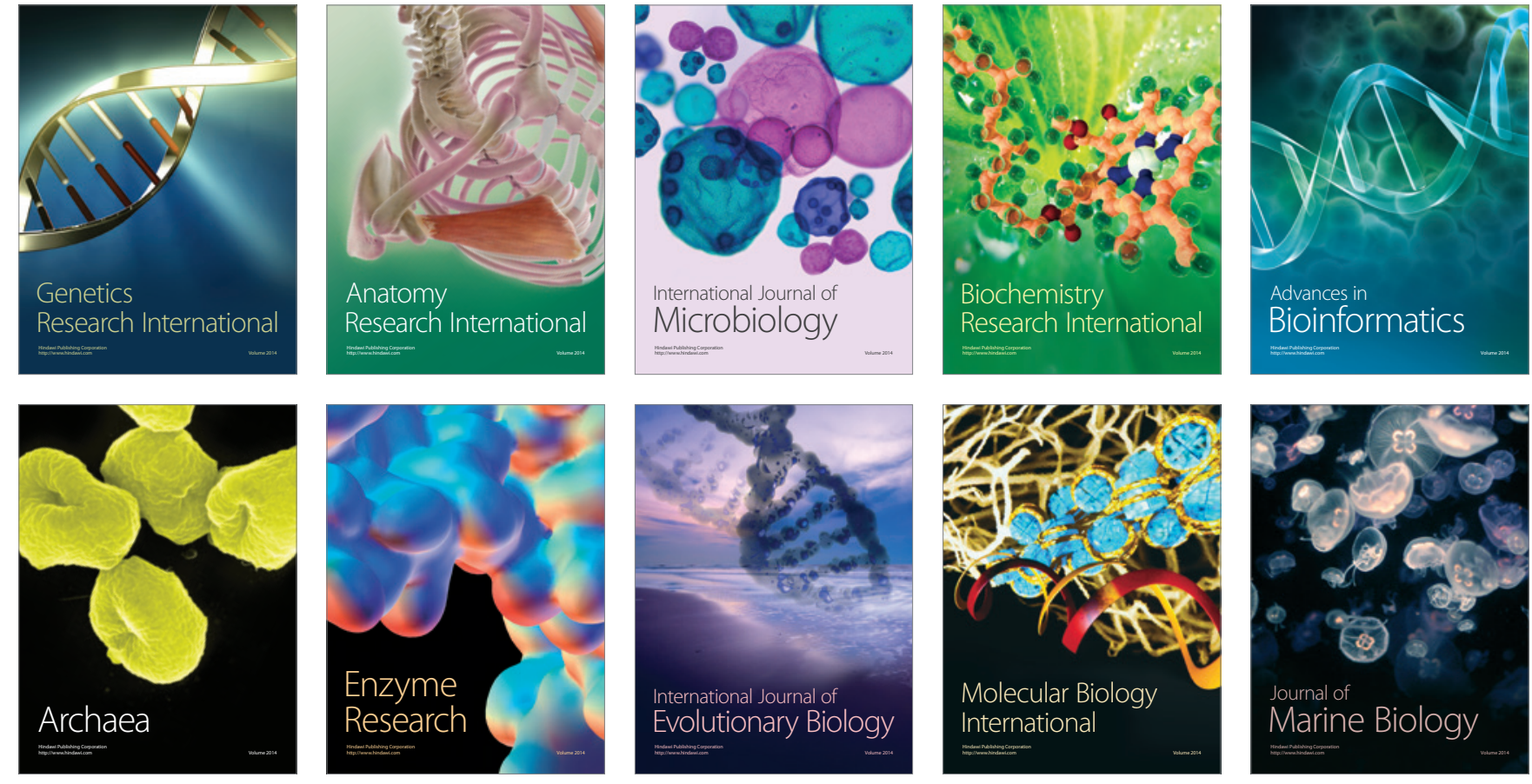